\title{
Interobserver Agreement in Vascular Invasion Scoring and the Added Value of Immunohistochemistry for Vascular Markers to Predict Disease Relapse in Stage I Testicular Nonseminomas
}

\author{
João Lobo, MD, *广ł\$ Hans Stoop, PhD,\| Ad J.M. Gillis, Ing, *\| \\ Leendert H.J. Looijenga, PhD, ${ }^{*} \|$ and Wolter Oosterhuis, MD, PhD\|
}

\begin{abstract}
Vascular invasion has been identified as an informative risk factor for relapse in stage I testicular nonseminomas, used to tailor treatment. We investigated interobserver agreement in vascular invasion reporting and studied the potential additional value of immunohistochemistry for vascular markers for predicting relapse. Patients $(n=52)$ with stage I testicular nonseminomas undergoing surveillance (1993-2006) were included (median follow-up of $66 \mathrm{mo}$ ). Two formalin-fixed paraffinembedded blocks with $>1 \mathrm{~cm}^{2}$ tissue and tumor/normal parenchyma interface were stained with hematoxylin and eosin and CD31, FVIII, and D2-40. Slides were assessed by 3 independent testicular germ cell tumor-dedicated pathologists, and agreement was assessed using Cohen $\kappa$ statistic. Sensitivity, specificity, and
\end{abstract}

From the *Princess Máxima Center for Pediatric Oncology, Heidelberglaan, Utrecht; \|Labaoratory for Experimental Patho-Oncology (LEPO), Department of Pathology, Erasmus MC-University Medical Center Rotterdam, Cancer Institute, Rotterdam, The Netherlands; $\dagger$ Department of Pathology, Portuguese Oncology Institute of Porto (IPOP), R. Dr. António Bernardino de Almeida; $\star$ Cancer Biology and Epigenetics Group, IPO Porto Research Center (GEBC CIIPOP), Portuguese Oncology Institute of Porto (IPO Porto) \& Porto Comprehensive Cancer Center (P.CCC), R. Dr. António Bernardino de Almeida; and §Department of Pathology and Molecular Immunology, Institute of Biomedical Sciences Abel Salazar, University of Porto (ICBAS-UP), Porto, Portugal.

L.H.J.L. and J.W.O. contributed equally to the work.

Conflicts of Interest and Source of Funding: J.L. is supported by FCTFundação para a Ciência e Tecnologia (POCI-01-0145-FEDER29043 and SFRH/BD/132751/2017). The authors have disclosed that they have no significant relationships with, or financial interest in, any commercial companies pertaining to this article.

Correspondence: Wolter Oosterhuis, $\mathrm{MD}, \mathrm{PhD}$, Laboratory for Experimental Patho-Oncology (LEPO), Department of Pathology, Erasmus MC-University Medical Center Rotterdam, Cancer Institute, 3000 CA Rotterdam, The Netherlands (e-mail: j.w.oosterhuis@erasmusmc.nl).

Supplemental Digital Content is available for this article. Direct URL citations appear in the printed text and are provided in the HTML and PDF versions of this article on the journal's website, www.ajsp. com.

Copyright (C 2019 The Author(s). Published by Wolters Kluwer Health, Inc. This is an open-access article distributed under the terms of the Creative Commons Attribution-Non Commercial-No Derivatives License 4.0 (CCBY-NC-ND), where it is permissible to download and share the work provided it is properly cited. The work cannot be changed in any way or used commercially without permission from the journal. accuracy of vascular invasion scoring in predicting relapse were calculated. Agreement among testicular germ cell tumordedicated pathologists was moderate $(\kappa=0.49$ to 0.54$)$, as was performance in predicting disease relapse (particularly, specificity of $86 \%$ ). Immunohistochemistry increased overall sensitivity $(71 \%)$, but decreased specificity $(71 \%)$ in predicting relapse. All patients $(n=8)$ with both blood and lymphatic vascular invasion developed a relapse. In multivariable analysis (including age, tumor size, rete testis invasion, and serum tumor markers), only vascular invasion had an independent impact in predicting relapse. Assessment of vascular invasion by testicular germ cell tumor-dedicated pathologists is good and is clinically meaningful, predicting disease relapse. Immunohistochemistry for vascular markers improves sensitivity of detecting disease relapse and allows for the identification of high-risk patients with both blood and lymphatic vascular invasion simultaneously, potentially of interest for tailored chemotherapy.

Key Words: immunohistochemistry, nonseminomas, relapse, testicular germ cell tumors, vascular invasion

(Am J Surg Pathol 2019;43:1711-1719)

$\mathrm{T}$ esticular germ cell tumors are the most common neoplasms among young-adult Caucasian men, and incidence has been rising in most countries. ${ }^{1}$ They represent, however, one of the most curable solid neoplasms, and current guidelines include the option of assigning both seminoma and nonseminoma stage I patients to active surveillance, a strategy that is being increasingly adopted as the preferred management modality. ${ }^{2}$ Indeed, about $70 \%$ to $85 \%$ of stage I testicular germ cell tumor patients ( $72 \%$ to $75 \%$ specifically for stage I nonseminoma patients according to published series) are cured with orchiectomy alone. ${ }^{3-5}$ Nevertheless, a group of patients ultimately develop disease relapse, particularly nonseminoma patients, and especially in the first 2 years after orchiectomy, implying additional treatment. ${ }^{6}$ Hence, biomarkers are urgently needed that can accurately predict which patients are going to experience relapse, so that treatment strategies can be individually tailored: avoiding unnecessary overtreatment of low-risk patients who can be maintained 
in surveillance, sparing them from the deleterious longterm side effects of cytotoxic therapies, and allowing for a better selection of patients who really benefit from adjuvant treatment after orchiectomy, so as to avoid disease relapse and associated complications. 7,8

Vascular invasion has been pointed out as such a marker in various studies, an idea first suggested by Raghavan et $\mathrm{al}^{9}$ and Peckam et al. ${ }^{10}$ The presence of vascular invasion is associated with poor prognosis, relapse, and metastases, particularly in nonseminomas. ${ }^{5,11-25}$ The relevance of vascular invasion in testicular germ cell tumors is illustrated by its inclusion in the TNM staging system, resulting in a pT2. ${ }^{26}$ Given this clinical importance, strict criteria for establishing the diagnosis of vascular invasion are recommended by expert panels, as some representations may result in overdiagnosis and, hence, possible overtreatment of patients. 2,24,27 However, testicular germ cell tumors are overall infrequent and, at the same time, are among the most diverse tumors in human pathology. This heterogeneity is problematic, as pathologists may see few cases a year in their routine, leaving room for disagreements in clinically relevant areas, which can lead to overtreatment. ${ }^{28,29}$ Studies have reported discrepancies in the pathologic assessment of testicular germ cell tumors between peripheral, general pathology departments, and centers with more expertise and dedication to these tumors. In fact, the most significant disagreement between these kinds of centers relates to vascular invasion interpretation (especially in nonseminoma cases), putting in evidence that the reproducibility on reporting this parameter by pathologists is far from ideal. ${ }^{30-32}$

In current guidelines, there is no formal indication for staining with vascular markers in order to aid the detection of vascular invasion, nor is it mandatory to discriminate the type of vessel invasion present in a tumor (either lymph vessel invasion, blood vessel invasion, or both). ${ }^{24,33}$ However, studies dealing with this matter in testicular germ cell tumors are lacking, and this was stated as the reason for not establishing a recommendation on the topic in the last International Society of Urological Pathology (ISUP) Testicular Cancer Consultation Cancer Recommendations meeting. $^{24}$

In this work, we aim to assess the inter-rater agreement between testicular germ cell tumor-dedicated pathologists in reporting vascular invasion. We also aim to determine the clinical value of adding immunohistochemistry for vascular markers to routine hematoxylin and eosin (H\&E) when carrying out this assessment for ultimately predicting disease relapse in a cohort of stage I nonseminoma patients, all undergoing surveillance. Finally, we intend to confirm the prognostic role of vascular invasion, namely its impact on relapse-free survival, and to explore the additional value of knowing the type of vessel invaded by the tumor.

\section{MATERIALS AND METHODS}

\section{Patients and Samples}

All patients consecutively diagnosed with stage I testicular nonseminoma between 1993 and 2006 were retrospectively queried from our database (which includes patients submitted to orchiectomy in various centers of the Netherlands). From these, only patients who underwent a surveillance strategy after orchiectomy were included in the study (ie, all patients undergoing adjuvant treatments were excluded). Clinical charts of each patient were reviewed by an investigator blinded to the results of the analysis. Variables collected included the following: age and serum tumor markers at diagnosis; tumor histologic subtype, laterality, size, multifocality, presence of vascular invasion, and rete testis invasion (according to the original pathology report of the respective center); therapies used for relapses (surgery and chemotherapy); type and topography of relapses; and dates of birth, diagnosis, relapse, death, and last follow-up. Disease relapse was defined as "early" or "late," as defined elsewhere. ${ }^{34}$ Patients without the required clinical information were excluded.

Orchiectomy samples were fixed overnight in $10 \%$ buffered formalin and paraffin-embedded. As regards tissue sample selection, it was determined that all cases should have at least 2 blocks with tumor representation available; that at least one of these blocks (preferably both) contained the interface between tumor and adjacent testicular parenchyma; and that each block contained at least $1 \mathrm{~cm}^{2}$ of tissue. Accompanying $\mathrm{H} \& \mathrm{E}$ slides were scanned for selecting sections fulfilling these criteria. Four serial 4- $\mu \mathrm{m}$ sections were then ordered from samples complying to these criteria, one for H\&E staining and the remaining for immunohistochemistry.

\section{Immunohistochemistry}

Immunohistochemistry was performed using the polymer detection method. After deparaffinization, antigen retrieval was performed in a pressure cooker after pretreatment with TRIS-EDTA at $\mathrm{pH}$ 9. Endogenous peroxidase activity was blocked by $3 \%$ hydrogen peroxide. Sections were then blocked for endogenous biotin with the Vector blocking kit. Slides were incubated overnight with anti-D2-40 antibody (1:100, clone D2-40; Dako, Glostrup, Denmark), anti-CD31 antibody (1:50, clone JC70A; Dako), and anti-factor 8 antibody (1:8000, polyclonal; Dako). Antigen-antibody complexes were detected using the Vector Avidin Biotin HRP, and visualization was performed with 3,3'-Diaminobenzidine and hydrogen peroxide. Hematoxylin was used for counterstaining for 3 minutes. Vessels of the tissue were used as (internal) positive control.

\section{Vascular Invasion Assessment}

Vascular invasion was assessed on H\&E slides (without access to immunostaining) by 3 independent testicular germ cell tumor-dedicated pathologists (Klaas van Kerckhoven, J.L., and a renowned expert, W.O.). The exact same set of slides was presented to each observer, and their assessment was independent and blinded to any clinicopathologic data. All pathologists rated vascular invasion as a dichotomic variable: "without vascular invasion" versus "with vascular invasion." In a later phase, discordant cases among the 3 testicular germ cell tumordedicated pathologists were further discussed in group under the microscope until a consensus was met. 
Immunohistochemistry evaluation of the cases was performed by J.W.O., again independently and blinded to any clinicopathologic data. Besides scoring vascular invasion as "absent" or "present," the type of vessel composing the vascular invasion was also discriminated: lymph vessel, blood vessel, or presence of both. The antibody panel was used as indicated: D2-40 for specifically staining lymph vessel endothelium; CD31 and Factor VIII both for staining blood vessel endothelium, and also for weakly staining lymph vessels.

\section{Statistical Analysis}

The data were tabulated using Microsoft Excel 2016 and analyzed using IBM SPSS Statistics version 24. Percentages were calculated on the basis of the number of cases with available data. Cohen $\kappa$ was run to determine whether there was agreement between 2 pathologists' judgment on whether vascular invasion was present on the available $\mathrm{H} \& \mathrm{E}$ slides. The level of agreement was categorized as in the guidelines by Landis and Koch. ${ }^{35}$ Associations between categorical variables were assessed using $\chi^{2}$ (with continuity correction). Distribution of continuous variables among groups was compared using the nonparametric Mann-Whitney $U$ test. Sensitivity, specificity, positive predictive value, negative predictive value, and accuracy of vascular invasion assessment on predicting relapse was calculated. Survival analyses were computed with Kaplan-Meier estimator and log-rank test. Hazard ratios and respective $95 \%$ confidence intervals were estimated using Cox regression models. A $P$-value equal or inferior to 0.05 was considered statistically significant.

\section{RESULTS}

\section{Cohort Characterization}

A total of 52 patients met the study criteria and were included. Detailed clinicopathologic features of the patients are depicted in Table 1. Median follow-up time was 66 months (interquartile range: 40 to $93 \mathrm{mo}$ ). Median patients' age at diagnosis was 31 years (interquartile range: 24 to $35 \mathrm{y}$ ). Seven tumors showed pure histology (5 pure embryonal carcinomas and 2 pure postpubertal-type teratomas), and $45(86.6 \%)$ were mixed tumors. Thirty-one $(59.6 \%)$ patients experienced disease relapse, only 3 of them corresponding to late relapses (at 65 , 48 , and just over $24 \mathrm{mo}$ ). One of the patients with late relapse died from disease at 63 months (with metastatic dissemination to liver, lung, and retroperitoneal lymph nodes), and another one died because of an intercurrence unrelated to his disease at 31 months. Patients with relapse received chemotherapy, and additional retroperitoneal lymph node dissection in the case of 5 patients. Two of the patients developed a second relapse, for which they received further treatment. Detailed information about histologic components and treatment of relapses is depicted in Supplemental Digital Content 1 (http://links.lww. com/PAS/A834).

Vascular invasion, as considered in the final pathology report, was significantly associated with relapse $(P<0.001)$. No significant association was depicted between any other variable (patients' age, elevation of serum tumor markers, histology, rete testis invasion, or tumor size) and the event of disease relapse. In our cohort,
TABLE 1. Clinicopathologic Features of Stage I Nonseminoma Patients

\begin{tabular}{|c|c|}
\hline Variables & $\begin{array}{l}\text { Patient Cohort }(\mathrm{N}=52), \\
\mathrm{n} / \mathrm{N}(\%)\end{array}$ \\
\hline Age (median, interquartile range) (y) & $31(24-35)$ \\
\hline \multicolumn{2}{|l|}{ Laterality } \\
\hline Right & $21 / 52(40.4)$ \\
\hline Left & $31 / 52(59.6)$ \\
\hline \multicolumn{2}{|l|}{ Preoperative serum tumor markers } \\
\hline Within normal range & $20 / 52(38.5)$ \\
\hline Elevated & $32 / 52(61.5)$ \\
\hline \multicolumn{2}{|l|}{ Histologic subtypes* } \\
\hline Pure embryonal carcinoma & $5 / 52(9.6)$ \\
\hline Pure postpubertal-type teratoma & $2 / 52(3.8)$ \\
\hline Mixed tumor, without seminoma & $21 / 52(40.4)$ \\
\hline $\begin{array}{l}\text { Mixed tumor, with seminoma } \\
\text { (combined tumor) }\end{array}$ & $24 / 52(46.2)$ \\
\hline \multicolumn{2}{|l|}{ Multifocality* } \\
\hline Absent & $50 / 52(96.2)$ \\
\hline Present & $2 / 52(3.8)$ \\
\hline $\begin{array}{l}\text { Largest tumor size (median, interquartile } \\
\text { range)*(cm) }\end{array}$ & $3.5(2.5-5.4)$ \\
\hline \multicolumn{2}{|l|}{ Rete testis invasion* } \\
\hline Absent & $30 / 42(71.4)$ \\
\hline Present, stromal & $9 / 42(21.4)$ \\
\hline Only pagetoid spread of GCNIS & $3 / 42(7.1)$ \\
\hline \multicolumn{2}{|l|}{ Vascular invasion* } \\
\hline Absent & $25 / 50(50.0)$ \\
\hline Present & $25 / 50(50.0)$ \\
\hline \multicolumn{2}{|l|}{ Relapse } \\
\hline No & $21 / 52(40.4)$ \\
\hline Yes & $31 / 52(59.6)$ \\
\hline \multicolumn{2}{|l|}{ Type of relapse } \\
\hline Early & $28 / 31(90.3)$ \\
\hline Late & $3 / 31(9.7)$ \\
\hline \multicolumn{2}{|l|}{ Site of relapse } \\
\hline Only serum markers & 6/31 (19.4) \\
\hline Serum markers+PAoLN & $14 / 31(45.2)$ \\
\hline Only PAoLN & $4 / 31(12.9)$ \\
\hline Only lung & $2 / 31(6.5)$ \\
\hline Serum markers+lung+PAoLN & $4 / 31(12.9)$ \\
\hline Serum markers+liver+lung+PAoLN & $1 / 31(3.2)$ \\
\hline \multicolumn{2}{|l|}{ Treatment performed for relapses } \\
\hline Only chemotherapy & 26/31 (83.9) \\
\hline Chemotherapy+RPLND & $5 / 31(16.1)$ \\
\hline \multicolumn{2}{|l|}{ Vital status at last follow-up } \\
\hline A-NED & $50 / 52(96.2)$ \\
\hline D-NED & $1 / 52(1.9)$ \\
\hline DFD & $1 / 52(1.9)$ \\
\hline
\end{tabular}

*According to the original pathology report.

A-NED indicates alive with no evidence of disease; DFD, died from disease; D-NED, died with no evidence of disease; GCNIS, germ cell neoplasia in situ; PAoLN, para-aortic lymph nodes; RPLND, retroperitoneal lymph node dissection.

patients with the presence of embryonal carcinoma did not show a significantly worse relapse-free survival compared with patients without the presence of embryonal carcinoma. Moreover, no significant association was depicted between tumor size and rete testis invasion, between rete testis invasion and vascular invasion, or between tumor size and vascular invasion.

\section{Interobserver Agreement in Vascular Invasion Scoring on $\mathrm{H} \& \mathrm{E}$}

A moderate agreement was always found with regard to the scoring of vascular invasion by the 3 testicular 
TABLE 2. Interobserver Agreement in Vascular Invasion Scoring

\begin{tabular}{|c|c|c|}
\hline \multicolumn{3}{|c|}{$\begin{array}{l}\text { Testicular Germ Cell Tumor-dedicated Pathologists' Assessment } \\
\text { of Vascular Invasion }\end{array}$} \\
\hline & Absent & Present \\
\hline $\begin{array}{l}\text { Agreement: Cohen } \kappa=0.54 \\
\qquad(P<0.001)\end{array}$ & \multicolumn{2}{|c|}{ Pathologist 3} \\
\hline \multicolumn{3}{|l|}{ Pathologist 1} \\
\hline Absent & 23 & 4 \\
\hline Present & 8 & 17 \\
\hline $\begin{array}{l}\text { Agreement: Cohen } \kappa=0.50 \\
\qquad(P<0.001)\end{array}$ & \multicolumn{2}{|c|}{ Pathologist 2} \\
\hline \multicolumn{3}{|l|}{ Pathologist 1} \\
\hline Absent & 22 & 5 \\
\hline Present & 8 & 17 \\
\hline $\begin{array}{l}\text { Agreement: Cohen } \kappa=0.49 \\
\quad(P<0.001)\end{array}$ & \multicolumn{2}{|c|}{ Pathologist 2} \\
\hline Pathologist 3 & & \\
\hline Absent & 24 & 7 \\
\hline Present & 6 & 15 \\
\hline
\end{tabular}

germ cell tumor-dedicated pathologists $(\kappa=0.49,0.50$, $0.54, P<0.001$ for all). The pairs of observers were in agreement in $75 \%$ to $80 \%$ of the times (Table 2 ).

\section{Performance of Vascular Invasion Scoring in Predicting Disease Relapse}

The independent H\&E assessment of vascular invasion by the 3 testicular germ cell tumor-dedicated pathologists always associated with disease relapse $(P=0.001,0.009,0.012)$. When judging the consensus of the 3 testicular germ cell tumor-dedicated pathologists (on H\&E only) as the "standard," the following performance on predicting relapse was achieved: sensitivity $=61.3 \%$, specificity $=85.7 \%$, positive predictive value $=86.4 \%$, negative predictive value $=60.0 \%$, and accuracy $=71.2 \%$ (Table 3). Of the 22 cases wherein H\&E assessment determined there was vascular invasion, $19(86.4 \%)$ indeed developed relapse. However, 12 patients in whom vascular invasion was reported as absent $(40.0 \%)$ developed relapse (Table 4).

By adding immunohistochemistry for the aforementioned vascular markers, sensitivity in predicting disease relapse was increased $(71.0 \%)$, and specificity was decreased (71.4\%), maintaining the same accuracy (Table 3). Immunohistochemistry assessment upgraded 8

TABLE 3. Performance Parameters for Predicting Disease Relapse on the Basis of Vascular Invasion Scoring

\begin{tabular}{lccccc}
\hline $\begin{array}{l}\text { Vascular } \\
\text { Invasion }\end{array}$ & $\begin{array}{c}\text { Sensitivity } \\
\mathbf{( \% )}\end{array}$ & $\begin{array}{c}\text { Specificity } \\
\mathbf{( \% )}\end{array}$ & $\begin{array}{c}\text { PPV } \\
\mathbf{( \% )}\end{array}$ & $\begin{array}{c}\text { NPV } \\
\mathbf{( \% )}\end{array}$ & $\begin{array}{c}\text { Accuracy } \\
\mathbf{( \% )}\end{array}$ \\
\hline Hcoring & 61.3 & 85.7 & 86.4 & 60.0 & 71.2 \\
Imm (consensus) \\
$\begin{array}{l}\text { chemistry } \\
\text { (D2-40+FVIII }\end{array}$ & 71.0 & 71.4 & 78.6 & 62.5 & 71.2 \\
$\quad$ CD31) & & & & \\
\hline & & & & \\
\hline
\end{tabular}

TABLE 4. Association Between Vascular Invasion Scoring, Type of Vessel With Invasion, and Relapse

\begin{tabular}{lcc}
\hline Vascular Invasion Scoring & No Relapse (n) & Relapse (n) \\
\hline H\&E consensus & & \\
$\quad$ Without VI & 18 & 12 \\
$\quad$ With VI & 3 & 19 \\
IHC & 15 & \\
Without VI & 6 & 22 \\
$\quad$ With VI & 3 & 12 \\
IHC showing LVI only & 3 & 2 \\
IHC showing BVI only & 0 & 8 \\
IHC showing LVI+BVI & & \\
\hline
\end{tabular}

BVI indicates blood vessel invasion; IHC, immunohistochemistry; LVI, lymph vessel invasion; VI, vascular invasion.

cases of absent vascular invasion on $H \& E$ to presence of vascular invasion, and 3 of these patients developed relapse. Moreover, it downgraded 2 cases of presence of vascular invasion on $\mathrm{H} \& \mathrm{E}$ to absence of vascular invasion, and these patients did not develop relapse.

Immunohistochemistry allowed for determination of 15 patients with lymph vessel invasion only, of whom 12 developed relapse $(80.0 \%) ; 5$ patients showing blood vessel invasion only, of whom 2 developed relapse $(40.0 \%$; late relapse in both cases); and 8 patients with both lymph vessel and blood vessel invasion evidence, all of them showing disease relapse (Table 4). The vascular-invasive histotype was embryonal carcinoma in all cases except 2, showing seminoma in lymph vessels. Of the 13 patients who exhibited blood vessel invasion, $3(23.1 \%)$ showed relapse in a visceral organ; of the 23 patients who exhibited lymph vessel invasion, $15(65.2 \%)$ suffered relapse in lymph nodes. One of the patients with a second relapse showed both lymph vessel and blood vessel invasion.

Illustrative examples of vascular invasion patterns detected on immunohistochemistry, but missed on H\&E, and also a common vascular invasion mimicker, are depicted in Figures 1 and 2, respectively.

\section{Survival Analysis}

Patients classified as having a vascular invasion on the H\&E consensus assessment showed significantly worse relapse-free survival when compared with patients without vascular invasion $(P=0.001$, Fig. 3). In multivariable analysis (including age, tumor size, rete testis invasion, and serum tumor markers), vascular invasion showed an independent impact in predicting relapse (hazard ratio, 3.163; 95\% confidence interval, 1.31-7.63).

\section{DISCUSSION}

In the last decades, much effort has been put toward better stratifying stage I nonseminoma patients according to their risk of relapse. Specifically, the aim is to better discriminate those patients who truly benefit from further treatment after orchiectomy from those who can safely follow a surveillance protocol. ${ }^{6,37,38}$ The most consistent histological parameter to predict disease recurrence in clinical stage I nonseminoma has been vascular invasion, ${ }^{11-23}$ 

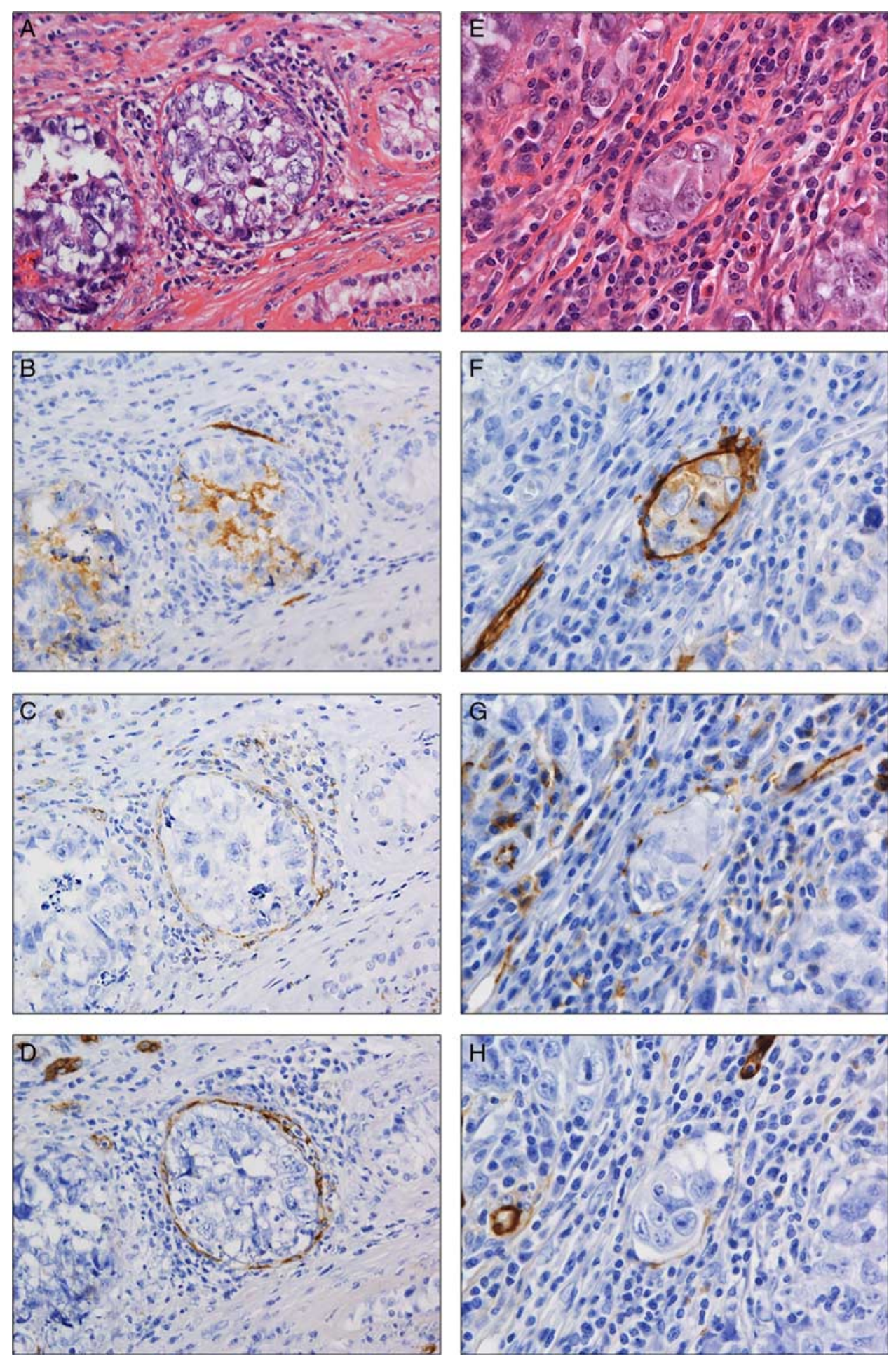

FIGURE 1. Illustrative examples of vascular invasion detected on immunohistochemistry that were missed on $H \& E$. A-D, A case of blood vessel invasion by embryonal carcinoma (left, top to bottom: H\&E, D2-40, CD31, and FVIII). E-H, A case of lymph vessel invasion by embryonal carcinoma (right, top to bottom: H\&E, D2-40, CD31, and FVIII). Part of this figure was also included by the authors in Moch et al. ${ }^{36}$

and our study was no exception, with vascular invasion being the only variable with significant impact on relapsefree survival after multivariable analysis. ${ }^{39,40}$ However, more factors apart from vascular invasion have been collected that demonstrate an impact in determining disease relapse, namely the amount of embryonal carcinoma. ${ }^{17}$ The 



FIGURE 2. Intratubular embryonal carcinoma as a mimicker of vascular invasion. Note the presence of necrosis and absence of clear staining for vascular markers (A-D: H\&E, D2-40, CD31, and FVIII). Part of this figure was also included by the authors in Moch et al. ${ }^{36}$

latter has been gaining robustness as a prognostic factor in nonseminoma patients, ${ }^{41,42}$ and it is biologically plausible

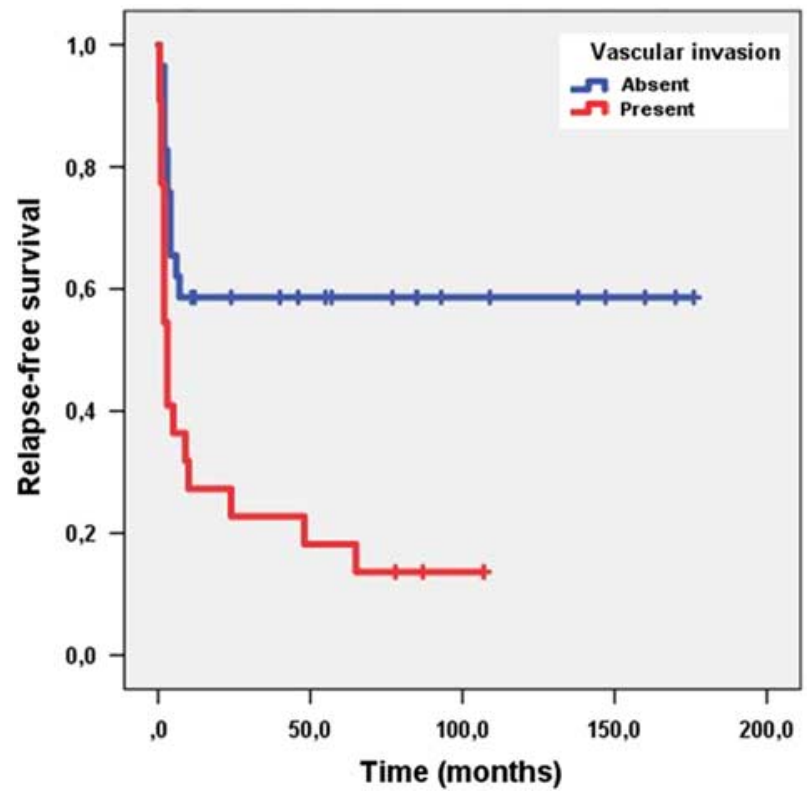

FIGURE 3. Relapse-free survival according to vascular invasion scoring. Tick marks in survival curves represent censored subjects over time.

that this is (at least partially) associated with vascular invasion itself. Despite that no recommendation is made to report the type of tumor responsible for vascular invasion in mixed tumors, ${ }^{24}$ our study demonstrates that almost all positive cases related to the embryonal carcinoma component (fitting with the model that metastatic cells are typically the cancer stem cells). ${ }^{36,43,44}$

Studies have shown that the global agreement in testicular pathology assessment among testicular germ cell tumor-dedicated pathologists is good. However, some disagreements still persist in areas such as staging and reporting of histologic subtypes. ${ }^{45}$ Moreover, studies have demonstrated the impact of centralized review of testicular germ cell tumor cases by experienced centers, with changes in the original report of up to $28 \%$, alterations in prognosis assessment in $9 \%$ of patients, and a predicted impact on therapy of $6.5 \%$ of patients. ${ }^{32}$ The most common reason for disagreement in nonseminomas was the assessment of vascular invasion. ${ }^{31}$ Another study demonstrated only a slight concordance in vascular invasion assessment between the original and reviewed reports $(\kappa=0.16)$, with only the revised scoring associating with nodal status at retroperitoneal lymph node dissection. ${ }^{30}$ Our work is in line with these studies, with the agreement in vascular invasion scoring among 3 testicular germ cell tumordedicated pathologists being good. Importantly, it was also clinically relevant, associating with relapse-free survival (including in multivariable analysis) and showing a good performance (accuracy of $71.2 \%$ ) in predicting disease relapse on 2 available $H \& E$ slides only. This underscores the consistency and clinical value of vascular invasion assessment by pathologists with experience in the field of germ cell cancer. Our results support the 
recommendation for medical oncologists to request, whenever possible, a review of testicular germ cell tumor cases by an experienced pathologist seeing a minimum of 30 cases a year. $^{2}$

The assessment of vascular invasion in most centers is routinely performed on the H\&E slide. Various representations can mimic vascular invasion and lead to overdiagnosis. One of such is the presence of histiocytes inside vessels and also intratubular nonseminoma (which almost always corresponds to intratubular embryonal carcinoma, Fig. 2). ${ }^{24}$ Accurate recognition of true vascular invasion may be hampered by poor fixation and related smear artifacts. ${ }^{46,47}$ Scarce sampling of fragments representing the interface with adjacent testicular parenchyma and albuginea is also a limiting factor, as most of the vascular invasion is found in

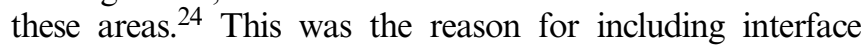
representation as an inclusion criterion of our study. Vascular invasion in testicular germ cell tumors is incorporated in the TNM staging system, representing pT2 disease (instead of pT1) in case of a positive finding, and subsequently, stage IB (instead of stage IA). Hence, pathologists are advised to follow strict criteria for defining it: tumor occupying a vascular structure delineated by flattened endothelial cells, in a cluster not conforming to the exact shape of the lumina; presence of accompanying fibrinous thrombosis, mural attachment, or reendothelialization; lack of obvious smear artifacts in nearby structures (such as lying on the tunical surface); and clusters that are more cohesive or smoothedged. 24,47 Experienced testicular germ cell tumor-dedicated pathologists are naturally aware of these criteria and follow them rigorously, and only score vascular invasion as present on $\mathrm{H} \& \mathrm{E}$ when absolutely sure, due to the known risk of overtreatment in case of a false-positive result (following the last ISUP recommendations). ${ }^{24}$ We hypothesize that this is the reason for the high specificity of $H \& E$ in predicting relapse. In contrast, most disagreements among testicular germ cell tumor-dedicated pathologists in our study occurred when the true expert (with decades of expertise) reported vascular invasion as present, whereas the 2 less experienced observers of the 3 considered it absent. We believe that these discordances in scoring might be due to difficult and challenging cases on H\&E, only recognized by an experienced pathologist.

There is, at the moment, no formal recommendation for performing immunohistochemistry studies for aiding in vascular invasion determination nor for the need to discriminate lymph vessel from blood vessel invasion. ${ }^{24}$ Few studies have assessed the latter, and it remains inconclusive whether discriminating among these might have additional value; one study has shown that only lymph vessel invasion associated with prognosis (metastases at diagnosis), ${ }^{48}$ while another paper reported the opposite scenario. ${ }^{49}$ Furthermore, a small pilot study on 24 testicular germ cell tumors showed that ERG immunohistochemistry could be of value in confirming vascular invasion, despite showing limited clinical impact in that small and heterogenous cohort of patients. ${ }^{50}$ We believe our work further extends these indications on the usefulness of immunohistochemistry. We have systematically performed immunohistochemistry for D240, Factor VIII, and CD31, ${ }^{51}$ and staining for these 3 markers resulted in increased sensitivity in predicting disease relapse $(71.0 \%)$, although at the expense of lower specificity (71.4\%), maintaining the same accuracy. The slight decrease in specificity for predicting relapse parallels the increase in sensitivity, and may result from misinterpretation of the staining due to artifacts. In practice, this allowed for the identification of 8 more cases of vascular invasion not depicted on H\&E, which showed clinical relevance for 3 of these patients, who ended up developing relapse. Two major tumor patterns emerged for explaining this discrepancy: the first one which exhibited vessels completely filled by solid masses of tumor cells without any discernable lumen; the second one which concerned small lymphatic vessels containing individual loose tumor cells, very hard to detect on H\&E without the aid of D240. In addition, immunohistochemistry avoided overscoring of vascular invasion in 2 cases when compared with H\&E interpretation, again with clinical relevance, as these patients did not develop relapse.

Moreover, immunohistochemistry allowed for discriminating lymph vessel from blood vessel invasion, the former being more frequent than the latter. This is biologically expected given the predominantly lymphatic route of metastases from testicular nonseminomas. We have identified 2 main patterns of blood vessel invasion: either large vessels with thickened walls, or vessels with faint, almost imperceptible walls, easily overlooked in H\&E staining, but clearly highlighted by CD31/Factor VIII. Importantly, it is noteworthy that all 8 cases presenting both with lymph vessel and blood vessel invasion experienced disease recurrence. This included, in fact, 1 patient who experienced a second relapse. This $100 \%$ accuracy in predicting recurrence when lymph vessel and blood vessel invasions are present in the same tumor may mean that these patients are better treated actively, instead of being referred for a surveillance strategy. The finding of these "double-vascular invasion" patients with a very high risk of recurrence has not been reported before, to the best of our knowledge.

One of the limitations of our work is its retrospective nature and the relatively small size of our cohort. Moreover, the amount of embryonal carcinoma in the tumor is lacking in our study, because it was not consistently reported in the original report. Futhermore, as stated above, we only examined 2 blocks of each case; hence, a true assessment of the totality of the tumors was not possible. Nevertheless, when looking at all cases considered not to display vascular invasion in the original report with access to all tumor material, 6 of them still showed vascular invasion in the 2 random blocks reviewed, putting in evidence the value of a centralized review even with access to limited material. In addition, our study has the strength of including a real "clean" cohort of only nonseminoma patients, all of whom had stage I disease and were undergoing a surveillance strategy. This way, we believe we have minimized the effect of confounder variables to the minimum and trust our results really reflect the value of vascular invasion scoring in predicting disease relapse. 
Moreover, patients were followed-up in different institutions, showing that the findings of this study are consistent in a heterogeneous population, and not due to a selection bias of only one niche of patients from a single center. Moreover, in this work, we verified the assumptions of the Cohen $\kappa$ test, so that it could be reliably used as a measure of interobserver agreement above the one expected just by chance.

All in all, we confirm that vascular invasion is indeed a major risk factor in stage I nonseminoma patients for predicting relapse, and that our scoring by testicular germ cell tumor-dedicated pathologists was accurate, consistent, and clinically relevant, even with access to $2 \mathrm{H} \& \mathrm{E}$ slides only, comparing with the performance reported by pathology referee panels. However, the interobserver agreement in scoring can be improved. The use of immunohistochemistry for this purpose, scarcely addressed in the literature and still a matter of debate due to insufficient data, shows additional value in detecting vascular invasion, with higher sensitivity in predicting relapse. We believe that, for the time being, careful examination of the tumor front and interface with parenchyma should be pursued, and, whenever there is a suspicious image of vascular invasion, the immunohistochemistry panel composed of CD31, Factor VIII, and D2-40 should definitely be ordered to confirm the findings (Supplemental Digital Content 2, http://links.lww.com/PAS/A835). However, remarkably, performing immunohistochemistry for these markers also allows for the identification of "lymph vessel plus blood vessel invasion" patients, which evidenced relapse in $100 \%$ of cases. This novel finding might allow discriminating a subgroup of "very high-risk" patients, who should probably be selected for further adjuvant treatment after orchiectomy. Hence, we believe our findings on additional use of immunohistochemistry might shed some light on the topic and potentiate future recommendations on it.

In our view, this and other works build up compelling evidence for the prognostic and predictive role of vascular invasion in nonseminoma patients. The stage is set, then, for establishing a proper prospective study on this matter, making use of a large, multicenter cohort of stage I nonseminoma patients to be differentially enrolled either in surveillance or adjuvant treatment according to vascular invasion scoring and characterization by immunohistochemistry. If the combined invasion of blood vessels and lymph vessels is confirmed in future prospective studies as a poor prognostic sign, we believe a recommendation for routinely performing these markers should be made.

\section{ACKNOWLEDGMENT}

The authors would like to acknowledge Klaas van Kerckhoven for the contribution to the work.

\section{REFERENCES}

1. Trabert B, Chen J, Devesa SS, et al. International patterns and trends in testicular cancer incidence, overall and by histologic subtype, 1973-2007. Andrology. 2015;3:4-12.

2. Honecker F, Aparicio J, Berney D, et al. ESMO Consensus Conference on testicular germ cell cancer: diagnosis, treatment and follow-up. Ann Oncol. 2018;29:1658-1686.
3. Nayan M, Hamilton RJ. Dissecting the evolving risk of relapse over time in surveillance for testicular cancer. Adv Urol. 2018;2018:7182014.

4. Kollmannsberger C, Moore C, Chi KN, et al. Non-risk-adapted surveillance for patients with stage I nonseminomatous testicular germ-cell tumors: diminishing treatment-related morbidity while maintaining efficacy. Ann Oncol. 2010;21:1296-1301.

5. Sturgeon JF, Moore MJ, Kakiashvili DM, et al. Non-risk-adapted surveillance in clinical stage I nonseminomatous germ cell tumors: the Princess Margaret Hospital's experience. Eur Urol. 2011;59:556-562.

6. Kollmannsberger C, Tandstad T, Bedard PL, et al. Patterns of relapse in patients with clinical stage I testicular cancer managed with active surveillance. J Clin Oncol. 2015;33:51-57.

7. Beyer J, Albers P, Altena R, et al. Maintaining success, reducing treatment burden, focusing on survivorship: highlights from the third European consensus conference on diagnosis and treatment of germcell cancer. Ann Oncol. 2013;24:878-888.

8. Curreri SA, Fung C, Beard CJ. Secondary malignant neoplasms in testicular cancer survivors. Urol Oncol. 2015;33:392-398.

9. Raghavan D, Peckham MJ, Heyderman E, et al. Prognostic factors in clinical stage I non-seminomatous germ-cell tumours of the testis. Br J Cancer. 1982;45:167-173.

10. Peckham MJ, Barrett A, Horwich A, et al. Orchiectomy alone for Stage I testicular non-seminoma. A progress report on the Royal Marsden Hospital study. Br J Urol. 1983;55:754-759.

11. Yilmaz A, Cheng T, Zhang J, et al. Testicular hilum and vascular invasion predict advanced clinical stage in nonseminomatous germ cell tumors. Mod Pathol. 2013;26:579-586.

12. Albers P, Siener R, Kliesch S, et al. Risk factors for relapse in clinical stage I nonseminomatous testicular germ cell tumors: results of the German Testicular Cancer Study Group Trial. J Clin Oncol. 2003;21: $1505-1512$.

13. Dong P, Liu ZW, Li XD, et al. Risk factors for relapse in patients with clinical stage I testicular nonseminomatous germ cell tumors. Med Oncol. 2013;30:494.

14. Costello AJ, Mortensen PH, Stillwell RG. Prognostic indicators for failure of surveillance management of stage I non-seminomatous germ cell tumours. Aust N Z J Surg. 1989;59:119-122.

15. Fung CY, Kalish LA, Brodsky GL, et al. Stage I nonseminomatous germ cell testicular tumor: prediction of metastatic potential by primary histopathology. J Clin Oncol. 1988;6:1467-1473.

16. Sonneveld DJ, Koops HS, Sleijfer DT, et al. Surgery versus surveillance in stage I non-seminoma testicular cancer. Semin Surg Oncol. 1999;17:230-239.

17. Daugaard G, Gundgaard MG, Mortensen MS, et al. Surveillance for stage I nonseminoma testicular cancer: outcomes and long-term follow-up in a population-based cohort. J Clin Oncol. 2014;32: 3817-3823.

18. Hoeltl W, Kosak D, Pont J, et al. Testicular cancer: prognostic implications of vascular invasion. J Urol. 1987;137:683-685.

19. Hoskin P, Dilly S, Easton D, et al. Prognostic factors in stage I nonseminomatous germ-cell testicular tumors managed by orchiectomy and surveillance: implications for adjuvant chemotherapy. $J$ Clin Oncol. 1986;4:1031-1036.

20. Javadpour N, Canning DA, O'Connell KJ, et al. Predictors of recurrent clinical stage I nonseminomatous testicular cancer. A prospective clinicopathologic study. Urology. 1986;27:508-511.

21. Klepp O, Olsson AM, Henrikson $\mathrm{H}$, et al. Prognostic factors in clinical stage I nonseminomatous germ cell tumors of the testis: multivariate analysis of a prospective multicenter study. SwedishNorwegian Testicular Cancer Group. J Clin Oncol. 1990;8:509-518.

22. Moriyama N, Daly JJ, Keating MA, et al. Vascular invasion as a prognosticator of metastatic disease in nonseminomatous germ cell tumors of the testis. Importance in "surveillance only" protocols. Cancer. 1985;56:2492-2498.

23. Sturgeon JF, Jewett MA, Alison RE, et al. Surveillance after orchidectomy for patients with clinical stage I nonseminomatous testis tumors. J Clin Oncol. 1992;10:564-568.

24. Verrill C, Yilmaz A, Srigley JR, et al. Reporting and staging of testicular germ cell tumors: the International Society of Urological Pathology (ISUP) Testicular Cancer Consultation Conference Recommendations. Am J Surg Pathol. 2017;41:e22-e32. 
25. Fujime M, Chang H, Lin CW, et al. Correlation of vascular invasion and metastasis in germ cell tumors of testis - a preliminary report. J Urol. 1984;131:1237-1241.

26. Amin MB, Edge S, Greene F, et al. AJCC Cancer Staging Manual, 8th ed. Chicago: Springer; 2017.

27. Ulbright TM. Pitfalls in the interpretation of specimens from patients with testicular tumours, with an emphasis on variant morphologies. Pathology. 2018:50:88-99.

28. Lobo J, Costa AL, Vilela-Salgueiro B, et al. Testicular germ cell tumors: revisiting a series in light of the new WHO classification and AJCC staging systems, focusing on challenges for pathologists. Hum Pathol. 2018;82:113-124.

29. Lobo J, Gillis AJM, Jeronimo C, et al. Human germ cell tumors are developmental cancers: impact of epigenetics on pathobiology and clinic. Int J Mol Sci. 2019:20.

30. Nicolai N, Colecchia M, Biasoni D, et al. Concordance and prediction ability of original and reviewed vascular invasion and other prognostic parameters of clinical stage I nonseminomatous germ cell testicular tumors after retroperitoneal lymph node dissection. J Urol. 2011;186:1298-1302.

31. Sharma P, Dhillon J, Agarwal G, et al. Disparities in interpretation of primary testicular germ cell tumor pathology. Am J Clin Pathol. 2015;144:289-294.

32. Purshouse K, Watson RA, Church DN, et al. Value of supraregional multidisciplinary review for the contemporary management of testicular tumors. Clin Genitourin Cancer. 2017;15:152-156.

33. Ulbright TM, Tickoo SK, Berney DM, et al. Best practices recommendations in the application of immunohistochemistry in testicular tumors: report from the International Society of Urological Pathology consensus conference. Am J Surg Pathol. 2014;38:e50-e59.

34. Dieckmann KP, Albers P, Classen J, et al. Late relapse of testicular germ cell neoplasms: a descriptive analysis of 122 cases. J Urol. 2005; 173:824-829.

35. Landis JR, Koch GG. The measurement of observer agreement for categorical data. Biometrics. 1977;33:159-174.

36. Moch H, Humphrey P, Ulbright T, et al. WHO Classification of Tumours of the Urinary System and Male Genital Organs, 4th ed. Lyon: IARC; 2016.

37. Mortensen MS, Lauritsen J, Kier MG, et al. Late relapses in stage I testicular cancer patients on surveillance. Eur Urol. 2016;70:365-371.

38. Shahidi M, Norman AR, Dearnaley DP, et al. Late recurrence in 1263 men with testicular germ cell tumors. Multivariate analysis of risk factors and implications for management. Cancer. 2002;95: $520-530$.
39. Warde P, Specht L, Horwich A, et al. Prognostic factors for relapse in stage I seminoma managed by surveillance: a pooled analysis. J Clin Oncol. 2002;20:4448-4452.

40. Kamba T, Kamoto T, Okubo K, et al. Outcome of different postorchiectomy management for stage I seminoma: Japanese multiinstitutional study including 425 patients. Int $J$ Urol. 2010;17: 980-987.

41. Heidenreich A, Sesterhenn IA, Mostofi FK, et al. Prognostic risk factors that identify patients with clinical stage I nonseminomatous germ cell tumors at low risk and high risk for metastasis. Cancer. 1998;83:1002-1011.

42. Lago-Hernandez CA, Feldman H, O'Donnell E, et al. A refined risk stratification scheme for clinical stage 1 NSGCT based on evaluation of both embryonal predominance and lymphovascular invasion. Ann Oncol. 2015;26:1396-1401.

43. Reya T, Morrison SJ, Clarke MF, et al. Stem cells, cancer, and cancer stem cells. Nature. 2001;414:105-111.

44. Cunningham JJ, Ulbright TM, Pera MF, et al. Lessons from human teratomas to guide development of safe stem cell therapies. Nat Biotechnol. 2012;30:849-857.

45. Berney DM, Algaba F, Amin M, et al. Handling and reporting of orchidectomy specimens with testicular cancer: areas of consensus and variation among 25 experts and 225 European pathologists. Histopathology. 2015;67:313-324.

46. Verrill C, Perry-Keene J, Srigley JR, et al. Intraoperative consultation and macroscopic handling: the International Society of Urological Pathology (ISUP) Testicular Cancer Consultation Conference Recommendations. Am J Surg Pathol. 2018;42:e33-e43.

47. French BL, Zynger DL. Do histopathologic variables affect the reporting of lymphovascular invasion in testicular germ cell tumors? Am J Clin Pathol. 2016;145:341-349.

48. Heinzelbecker J, Gross-Weege M, Weiss C, et al. Microvascular invasion of testicular nonseminomatous germ cell tumors: implications of separate evaluation of lymphatic and blood vessels. $J$ Urol. 2014;192:593-599.

49. Fukuda S, Shirahama T, Imazono Y, et al. Expression of vascular endothelial growth factor in patients with testicular germ cell tumors as an indicator of metastatic disease. Cancer. 1999;85:1323-1330.

50. Udager AM, McHugh JB, Morgan TM, et al. Utility of ERG immunohistochemistry for evaluation of lymphovascular invasion in testicular germ cell tumors: a retrospective pilot study. Appl Immunohistochem Mol Morphol. 2017;27:392-401.

51. Ye H, Ulbright TM. Difficult differential diagnoses in testicular pathology. Arch Pathol Lab Med. 2012;136:435-446. 